Development of a New Materials Platform to Study Iron and Diamond at Ultrahigh Pressures Using NIF

T. Duffy

December 19, 2012 
This document was prepared as an account of work sponsored by an agency of the United States government. Neither the United States government nor Lawrence Livermore National Security, LLC, nor any of their employees makes any warranty, expressed or implied, or assumes any legal liability or responsibility for the accuracy, completeness, or usefulness of any information, apparatus, product, or process disclosed, or represents that its use would not infringe privately owned rights. Reference herein to any specific commercial product, process, or service by trade name, trademark, manufacturer, or otherwise does not necessarily constitute or imply its endorsement, recommendation, or favoring by the United States government or Lawrence Livermore National Security, LLC. The views and opinions of authors expressed herein do not necessarily state or reflect those of the United States government or Lawrence Livermore National Security, LLC, and shall not be used for advertising or product endorsement purposes.

This work performed under the auspices of the U.S. Department of Energy by Lawrence Livermore National Laboratory under Contract DE-AC52-07NA27344. 
National Ignition Facility Concept Development Proposal - Final Report

Subcontract Number: $B 595803$

\title{
Development of a New Materials Platform to Study Iron and Diamond at Ultrahigh Pressures Using NIF
}

\author{
Thomas Duffy \\ Department of Geosciences \\ Princeton University \\ Princeton, NJ 08544
}

\section{Introduction}

The goal of this proposal was to develop the capability to subject solids to ultrahigh compression using ramp loading techniques at NIF. Specifically, we proposed to examine the equation of state, strength, and phase transitions in iron and diamond to 30 Mbar pressures. Diamond and iron are fundamental materials of profound interest in condensed matter physics, materials science, and planetary science. The behavior of these solids under ultrahigh compression is essential to understanding planetary interiors and for probing the limits to our understanding of solid state behavior at extreme conditions

\section{Background}

In thermodynamics, compression pathways are typically considered under conditions of either constant temperature (isothermal) or constant entropy (isentropic). The diamond-anvil cell provides an experimental tool for achieving high compressions under static, isothermal conditions, but capabilities for isentropic compression under controlled laboratory conditions are limited. In this project, we have worked toward achieving "quasi-isentropic" compression to the multi-TPa regime ( 1 terrapascal $=10$ million atmospheres), pressures previously unprecedented in such laboratory experiments.

Static compression experiments are, so far, limited to peak pressures of $\sim 0.3-0.6 \mathrm{TPa}$. In contrast, dynamic compression methods have reached the $100 \mathrm{TPa}(\sim 1$ billion atmosphere) level, albeit using shock wave techniques that are accompanied by large amounts of entropy production and heating. This limits the ability of such measurements to characterize atomic-bonding forces over a broad range of inter-atomic separations. Isentropic compression, on the other hand, yields lower temperatures, and therefore higher densities can be achieved while remaining in the solid state. The high compressions attained in isentropic compression are also important for research into inertial confinement fusion.

In addition, isentropic compression is of interest in simulating the pressure-temperature $(P-T)$ conditions achieved inside large planets, a capability essential for understanding the 
structure and evolution of planets. The cores of Jupiter, Saturn, and many extra-solar planets likely contain material at pressures $(\mathrm{P})$ greater than $1 \mathrm{TPa}$ (10 Mbar). Planetary companions are now known to be common among main-sequence stars and hundreds of exoplanets have been discovered to date. As the fourth most abundant element in the cosmos, carbon is a key constituent of many planets. Ice-giant planets such as Neptune and Uranus contain large quantities of methane that decompose under high-pressure and temperature conditions, possibly forming diamond-rich layers in their interiors. In extrasolar planetary systems with high ratios of carbon to oxygen, interiors may be dominated not by silicates but by carbides and graphite/diamond. Possible detections of such planets (WASP-12b, 55 Cancri e) have been reported.

Iron is the dominant constituent of Earth's core, along with approximately 6-10 wt.\% lighter components in the outer core. Pressures range from $136 \mathrm{GPa}$ at the core-mantle boundary (2891-km depth) to $364 \mathrm{GPa}$ at Earth's center (6371 km depth), with the crystalline inner core being surrounded by the liquid outer core. While the inner core is only $1.6 \%$ of Earth's mass, it is thought to be nearly pure iron and it exhibits a number of unexplained properties, including elastic anisotropy, hemispherical dichotomy and radial variability. Solidification of the inner core is expected to help power the geodynamo that produces Earth's magnetic field in the outer core.

Theoretical calculations suggest that Fe will undergo ultra-high pressure transitions to a face-centered-cubic (fcc) phase and finally to a body-centered-tetragonal (bct) phase. These transformations are predicted to occur above $2 \mathrm{TPa}$ and may occur under conditions expected in the deep interior of a 10 Earth-mass terrestrial planet. Theoretical calculations of the melting curve of iron yield a Clapeyron slope steeper than the expected geotherm for a super-Earth exoplanet which suggests that the likelihood of a molten core decreases as the size of a terrestrial planet increases. Ramp-compression techniques are necessary to provide experimental constraints on these planetary properties under such extreme conditions.

\section{Observations}

\section{Ramp compression of iron}

We carried out preliminary ramp compression experiments on iron at NIF (shot N110524). The goal of this experiment was to ramp-compress Fe after it had been precompressed into the high-pressure $\varepsilon$-phase, by way of an initial steady shock. This shock state represents the initial conditions for subsequent ramp compression. Our targets consisted of a stepped iron sample formed by vapor deposition onto a chemical-vapor-deposition (CVD) diamond ablator $(80-\mu \mathrm{m}$ thick, 3-mm diameter). The iron targets had three steps so that we can record the pressure-wave transit across four different thicknesses for each experiment. The steps were formed using a mask which was shifted to different positions during the deposition process. The source material was high purity $(99.995 \%)$ Fe and was deposited in 70-100 $\mu$ m layers under 
conditions that yielded a grain size of $\sim 5 \mu \mathrm{m}$ in the stress loading direction and $\sim 1 \mu \mathrm{m}$ in-plane. Pole figure measurements showed that the Fe bcc crystal structure was orientated with the [110] axis in the growth (pressure loading) direction with random orientation in-plane. The samples were measured to be fully dense $\left(7.87 \mathrm{~g} / \mathrm{cm}^{3}\right)$ to within $0.6 \%$.

The diamond ablator and stepped iron targets were attached over a 3-mm aperture on the side of a Au hohlraum. The hohlraum had a diameter of $6 \mathrm{~mm}$, a length of $11 \mathrm{~mm}$, and a laser entrance hole $4.5 \mathrm{~mm}$ in diameter and was filled with $0.1 \mathrm{~atm}$. of neopentane $\left(\mathrm{C}_{5} \mathrm{H}_{12}\right)$ gas held in by $0.6-\mu \mathrm{m}$ thick polyimide windows covering the laser entrance holes. A $10 \mu \mathrm{m}$-thick Au layer between the diamond and the Fe served to shield the stepped sample from being preheated by radiation from the hohlraum. Detailed radiative-transport simulations confirm the effectiveness of the Au layer, with a predicted temperature rise of only $33 \mathrm{~K}$ due to $\mathrm{x}$-ray preheat. No evidence of preheat was observed in the VISAR or streaked optical pyrometry for any of our experiments.

The inner walls of the hohlraum were illuminated with 175 beams of the NIF with a combined energy up to $285 \mathrm{~kJ}$ at $351 \mathrm{~nm}$ in a $26 \mathrm{~ns}$ temporally ramped laser pulse (Fig. 1, inset). This generated a spatially uniform distribution of thermal $\mathrm{x}$-rays with a characteristic radiation temperature $T_{\text {rad}}$, which monotonically increased in time to a peak $T_{\text {rad }} \sim 173 \mathrm{eV}$. The ablation of carbon from hohlraum $\mathrm{x}$-rays drives a time-dependent ramp compression wave through the stepped sample.

The response of the sample to the compression wave is characterized by velocity interferometry (VISAR) which records the velocity of the compression wave, the material velocity behind the pressure wave, and the velocity of the free (back) surface. The VISAR diagnostic uses a line-focused 660 -nm laser beam to monitor a one-dimensional $\sim 1 \mathrm{~mm}$ region of the sample covering all four thicknesses. Changes in velocity of the diamond free surface produce phase shifts in interference fringes that are recorded with a streak camera. A typical VISAR data record had a 5 - $\mu \mathrm{m}$ spatial resolution, a 10-ns streak window with $0.01 \mathrm{~ns}$ time resolution, and minimum velocity per fringe of $5.46 \mathrm{~km} / \mathrm{s}$. The fringe position was determined to better than $2 \%$ of a fringe giving a velocity resolution of $0.1 \mathrm{~km} / \mathrm{s}$. A Lagrangian analysis is used to translate the wave profile measurements into a stress-density relation that describes the loading path of the sample

The result of this shot was that we successfully shock compressed all four iron steps with a steady shock to $65 \mathrm{GPa}$. Subsequently, a shock formed within the bulk Fe sample that caused the VISAR record to blank. However, successful VISAR signals were recorded for two of the four steps to pressures above 2 Mbar (Fig. 1). The kinetics of the $\alpha-\varepsilon$ phase transformation in iron was fast compared to the time resolution of the shot. Lagrangian analysis was performed to obtain stress-density states up to $240 \mathrm{GPa}$ from the measured wave profile. The results are in good agreement with earlier experiments on Omega, demonstrating the consistency of results obtained using these two facilities. The results of this experiment will also enable us to develop a refined and improved pulse shape for future ramp compression experiments on Fe at NIF. A more detailed description of our work on Fe is contained in a submitted manuscript (Wang et al., 2012). 


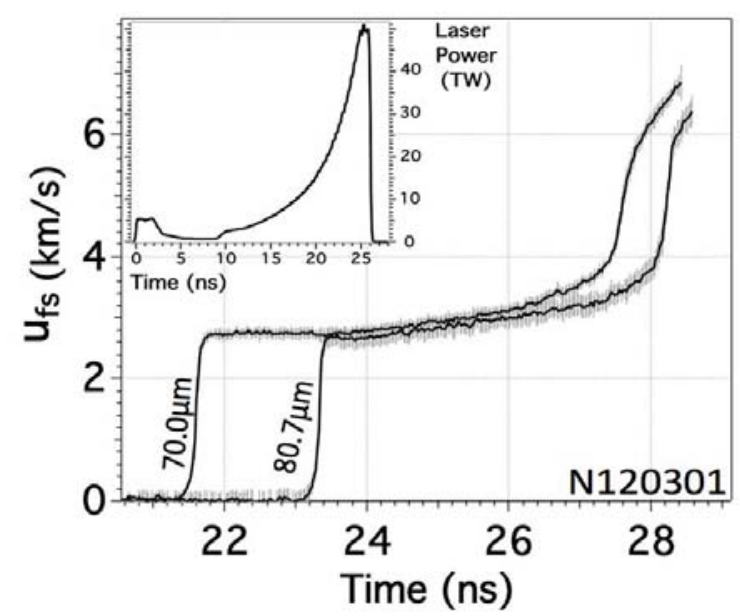

Fig. 1. NIF shot N120301. Inset: Laser pulse used at NIF. The first 8ns of the laser pulse was designed to launch a steady shock into the multi-step Fe sample. The free surface velocity profiles for the two thinnest steps are shown up to the level at which the VISAR blanked out.

\section{Ramp compression of carbon}

The goal of these experiments was to demonstrate the capability of achieving shock-less ("ramp") compression of carbon to $3 \mathrm{TPa}$ or more, pressures never before reached in dynamic experiments approaching isentropic loading. Our sample consists of synthetic diamond, shaped with three steps as in the case of the iron experiments described above. Our target assemblies consisted of a $50-\mu \mathrm{m}$ thick diamond plate used as an ablator, a 10- $\mu \mathrm{m}$ Au layer pre-heat shield and a diamond sample with four steps with thicknesses of 140/151.7/162.6/172.5 $\mu \mathrm{m}$ (Fig. 2 inset). The diamond samples were formed by chemical vapor deposition to yield a layered microstructure with an average grain size of $200 \mathrm{~nm}$ and a density of $3.25 \mathrm{~g} / \mathrm{cm}^{3}+/-0.01 \%$. The final sample had alternating $0.35 \mu \mathrm{m}$ layers of $20 \mathrm{~nm}$ grains followed by $\sim$ micron sized grains. Xray diffraction showed a $<110>$ texture in the growth direction. 176 beams of NIF at $351 \mathrm{~nm}$ with up to $0.76 \mathrm{MJ}$ of laser energy were focused on inner walls of an Au hohlraum to ramp compress the sample. Figure 2 shows the VISAR interferogram and resulting free surface velocity profile for shot N105161. The free surface particle velocity profiles, $\mathrm{u}_{\mathrm{fs}}(\mathrm{t})$ are characterized by an initial shock to $4.1 \mathrm{~km} / \mathrm{s}$, followed by a fast rise and plateau at $7.2 \mathrm{~km} / \mathrm{s}$ and subsequent ramp compression to $46.6 \mathrm{~km} / \mathrm{s}$. 


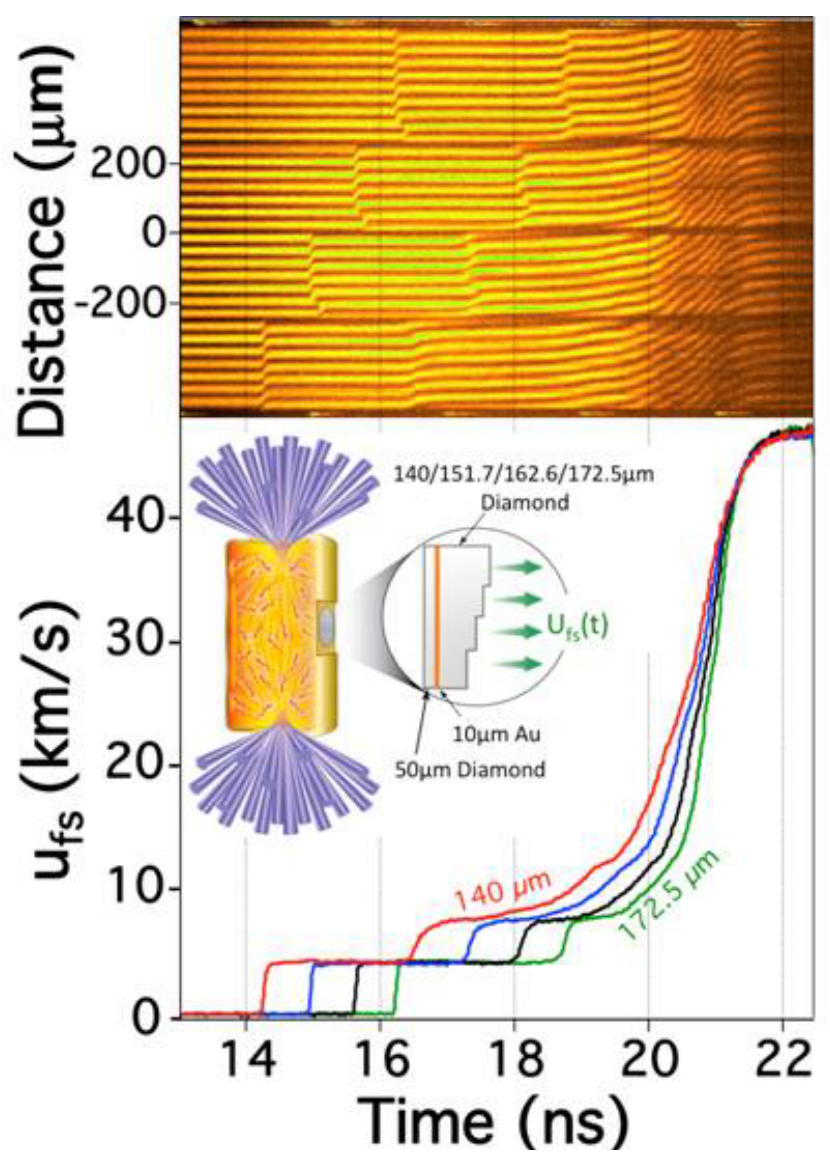

Fig. 2. Summary of NIF experiments on diamond sample (shot N105161). The upper panel shows an example of the VISAR interferogram recorded across all four thicknesses of the sample. The lower panel shows the recorded free surface velocity profiles. The inset shows a schematic illustration of the target platform.

An iterative Lagrangian analysis method was used to determine the Lagrangian sound speed $C_{\mathrm{L}}(\mathrm{u})$ and stress-density state, $P_{\mathrm{x}}-\rho$, from the measured $u_{\mathrm{fs}}(\mathrm{t})$ data (Fig. 3 ). In all, three shots giving $C_{\mathrm{L}}(\mathrm{u})$ and $P_{\mathrm{x}}-\rho$ data were conducted with peak stresses of 2.7, 3.7 and $5 \mathrm{TPa}$, respectively. The calculated stress-density response for the ramp-compressed diamond is shown as the red curve in Fig. 3 along with uncertainties represented by the light red band. Also plotted are shock Hugoniot data and previous ramp compression data from Omega to $0.8 \mathrm{TPa}$. Our ramp compression measurements are absolute (not referenced against a standard), and as there are no standards at TPa pressures, such absolute data are needed for determining equations of state and validating condensed-matter theory to the 1-100 TPa regime.

For comparison with our measurements, a Mie-Grüneisen EOS model is used to calculate an isentrope (dot-dashed brown curve) and a full density $\left(\rho_{0}=3.525 \mathrm{~g} / \mathrm{cm}^{3}\right)$ and porous Hugoniot $\left(\rho_{0}=3.25 \mathrm{~g} / \mathrm{cm}^{3}\right)$. Our data reveal a stress-density response intermediate between the isentrope and the Hugoniot. The diamond samples used in our experiments were measured to be $7.5 \%$ below full crystal density $\left(\rho_{0}=3.25 \mathrm{~g} / \mathrm{cm}^{3}\right)$. A number of studies on compression of under-dense 
materials have shown that rapid heating due to pore closure and the resultant increase in thermal pressure gives rise to reduced compressibility. In Fig. 2 this is witnessed by the stiffer response of the calculated porous Hugoniot compared to the Hugoniot for full-density diamond. Our ramp data are likewise expected to exhibit a stiffer response over ramp-compression of single crystal diamond, yet they do exhibit higher compressibility than the calculated $7.5 \%$ porous Hugoniot, indicative of a cooler compression path (lower thermal pressure). Still, our data reveal an offset relative to the calculated isentrope, suggesting that dissipation is present during ramp compression, albeit to a lesser degree than under shock loading.

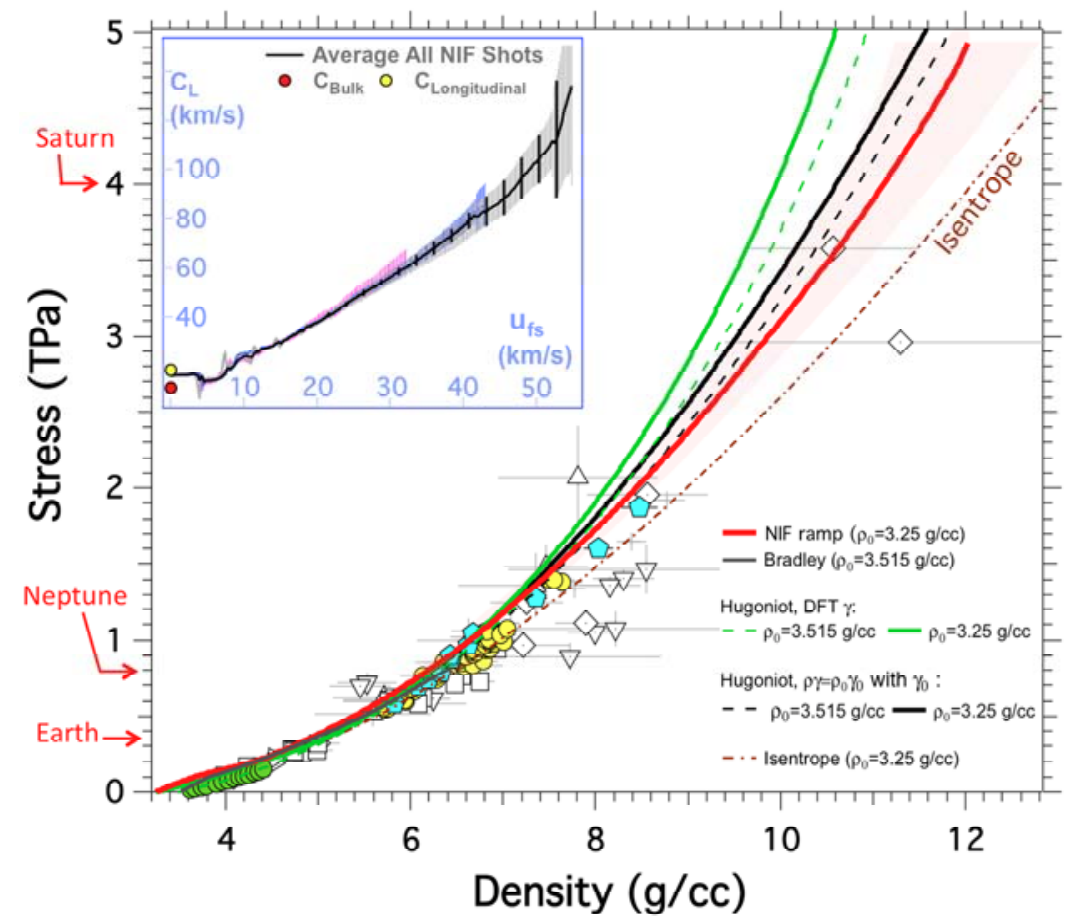

Fig. 3. Stress versus density for the NIF ramp data (red solid curve). For the same initial density the calculated Hugoniots (solid black and green curves) and isentrope are shown. Our data exhibits higher compressibility than the equivalent porous Hugoniot which is indicative of a cooler compression path. Also shown are calculated full density Hugoniots (dashed lines) and data from shock experiments and previous ramp-compression data from Omega. The predicted core pressures for Earth, Neptune and Saturn are indicated on the stress axis. Upper insert: Lagrangian sound speed versus free surface velocity for shots N110308, N110516, N110524 and the average of all three shots (black curve). 


\section{Conclusions}

In summary, we have demonstrated a new experimental technique to create material conditions consistent with those found in the centers of giant planets. Our stress of 5 TPa lies between the expected pressure at the center of Saturn (4 TPa) and Jupiter ( $7 \mathrm{TPa}$ ) where a core of heavy elements is expected to reside. At this pressure, diamond is compressed 3.7 times to a density of about $12 \mathrm{~g} / \mathrm{cm}^{3}$, greater than that of lead at ambient conditions. Future work will enable us to reach higher pressures (by using higher laser power) and make more detailed measurements of material properties through techniques such as x-ray diffraction and EXAFS. Future work will also focus on understanding how sample microstructure affects wave propagation response.

We have succeeded in obtaining high-quality equation of state data to $5 \mathrm{TPa}$, exceeding the target pressure for our project. While collecting this data we have also accumulated valuable information and experience related to the intersection of the laser pulse-shaping and target physics that will be highly useful in developing further high-pressure measurements on NIF. One

manuscript that includes NIF data has been submitted for publication. A second manuscript is in preparation and will be submitted in early 2013.

\section{Dissemination of Results}

Wang, J., R. F. Smith, J. H. Eggert, D. G. Braun, T. R. Boehly, J. R. Patterson, P. M. Celliers, R. Jeanloz, G. W. Collins, and T. S. Duffy, Ramp compression of iron to $273 \mathrm{GPa}$, Journal of Applied Physics, submitted, 2012.

Smith, R. F., J. H. Eggert, D. G. Braun, J. R. Patterson, R. E. Rudd, R. Jeanloz, T. S. Duffy, J. Biener, A. E. Lazicki, A. V. Hamza, J. Wang, T. Braun, L. X. Benedict, P. M. Celliers, and G. W. Collins, Diamond at $5000 \mathrm{GPa}$, in preparation, 2012.

Duffy, T. S., and N. Madhusudhan, Mineralogy of extra-solar planets, Treatise on Geophysics, in preparation, 2012. 